\title{
Verification of new model for determining the critical plane orientation angle
}

\author{
Marta Kurek $^{1, *}$ \\ ${ }^{1}$ Opole University of Technology, Mechanical Engineering Faculty, 5 Mikolajczyka Str., Opole, Poland
}

\begin{abstract}
The principal objective of this study is to establish a method which can enable the assessment of fatigue life at the stage of the design and building of machine parts and equipment. The paper contains a description of a manner in which the angle of a critical plane can be determined, which is coupled with its adequate verification. The study in the paper is based on an assumption of an idea of a critical plane which adopts that the occurrence of a fatigue crack of a material is caused by the occurrence of strain in the critical plane of a material. The conclusion from this study takes the form of a comparison between the experimental and calculated results performed by adoption of the developed model. The results demonstrate satisfactory level of conformity between the two.
\end{abstract}

\section{Introduction}

For over two hundred years the fatigue of structural materials has been the subject of interest to scholars and engineers. The subject of material fatigue is extensively studied and research due to the hazard of enormous material losses and safety risks that can be caused by a fatigue failure.

Many tragic catastrophes have been caused by fatigue cracks in construction elements, whose occurrence made it impossible for a load to be transferred. This, in turn, resulted in a complete failure of a structure. Another equally important aspect of material fatigue is the pursuit of reducing the mass and production costs as well as operation of machines and structures.

The material fatigue and construction phenomenon is a significant problem of our time. It appears in many branches of industry e.g. aerospace, machinery, mining and transport, and is especially visible in the case when an emergency or failure people results in deaths and injuries.

The main objective of the majority of research works [1-6] concerning the projected fatigue life is to find a method which can be applied for the estimation of the fatigue life already at the stage of the design and throughout the course of constructing elements of machines and equipment.

The aim of the work is to present a new model applicable for assessment of fatigue life on the basis of a concept of the critical plane accompanied by the analysis of the scatter of the results. Every model requires verification, best on the basis of experimental data.

\section{Multiaxial critical plane-based criterion}

Stress criteria for a significant group of the fatigue criteria can be derived from the classic damage theory. During their evolution a collection of criteria arose out of the concept of a critical plane. This group of criteria will be the main subject of this study. The critial plane concept assumes, that material fatigue cracking is caused by stress acting along the materials critical plane. The potential use of the critical plane in the research of multiaxial fatigue was first proposed by Stanfield in 1935 [7]. Today, there is a steadilly growing interest in this concept. In a paper datigng back to 2001 [8], the authors proposed a new multiaxial fatigue criterion, for which the angle of critical plane orientation is expressed using the sum of two angles. The first is the maximum angle defined by normal stresses, whereas the second one is angle $\beta$, whose value is expressed in relation to the direction given by the maximum in the normal direction and is expressed as

$$
\beta=\frac{3}{2}\left[1-\left(\frac{\tau_{a f}}{\sigma_{a f}}\right)^{2}\right] 45^{\circ}
$$

Several years later in literature [9] another proposition appeared as to how to express the given angle, i.e.:

$$
\beta_{1}=\frac{9}{8}\left[1-\left(\frac{\tau_{a f}}{\sigma_{a f}}\right)^{4}\right] 45^{\circ}
$$

\footnotetext{
Corresponding author: ma.kurek@po.opole.pl
} 


$$
\begin{aligned}
& \beta_{2}=\frac{3 \sqrt{3}}{3 \sqrt{3}-1}\left[1-\left(\frac{\tau_{a f}}{\sigma_{a f}}\right){ }^{3}\right] 45^{\circ} \\
& \beta_{3}=\frac{\sqrt{3}}{\sqrt{3}-1}\left[1-\left(\frac{\tau_{a f}}{\sigma_{a f}}\right)\right] 45^{\circ}, \\
& \beta_{4}=\frac{3}{(\sqrt{3}-1)^{2}}\left[1-\left(\frac{\tau_{a f}}{\sigma_{a f}}\right)\right]^{2} 45^{\circ}
\end{aligned}
$$

where: $\sigma_{a f}$ - fatigue limit for bending, $\tau_{a f}$ - fatigue limit for torsion

A graphical interpretation of the equations (1-5) is presented in Fig. 1. Depending on the adopted model the estimated fatigue life may vary considerably.

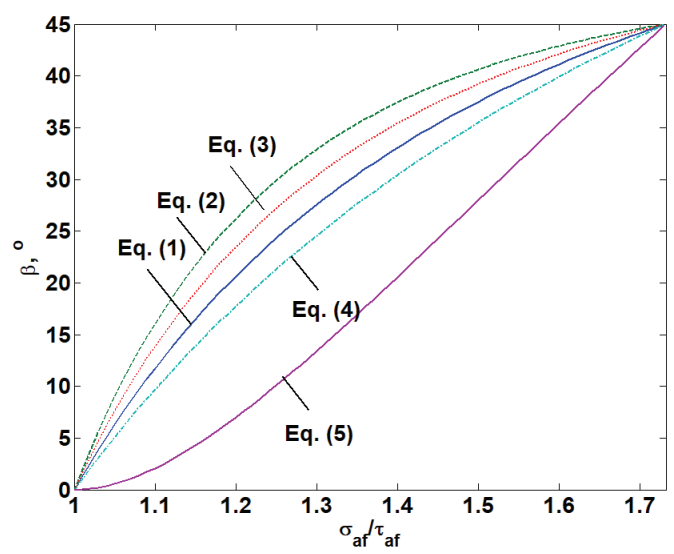

Fig. 1. Graphical representation of equations 1-5.

Each of the presented propositions refers in its expression to the fatigue life. It's worth noting that the equations (1-5) have a limited range. The adopted range is from 1 to $\sqrt{3}$. There is a number of various materials for which the ratio of the normal stress to the shear stress exceeds the range that is presented above.

Following the analysis of the variability of the calculated fatigue life that was performed in relation to the value of angle $\beta$ [10], a new expression was derived in the form:

$$
\operatorname{ctg}(4 \beta)=22.5\left[\left(\frac{1+\sqrt{3}}{2}\right)-\frac{\sigma_{a}\left(N_{f i}\right)}{\tau_{a}\left(N_{f i}\right)}\right]=22.5\left[\frac{1+\sqrt{3}}{2}-B_{2}\left(N_{f i}\right)\right],
$$

where:

$$
B_{2}\left(N_{f i}\right)=\frac{\sigma_{a}\left(N_{f i}\right)}{\tau_{a}\left(N_{f i}\right)} .
$$

A new relation presented below was developed on the basis of fatigue testing into several construction materials.

A graphical interpretation of formula (6) is given in Fig. 2 , whereas the broken line marks the proposition developed by Carpinteri (1).

In its current form, the newly proposed relation (formula 6) accounts for the ratio of normal to shear stresses. This forms a significant novelty in relation to the earlier statements, which involve the ratios of the fatigue boundaries.

For a variety of materials, in particular the ones with non-parallel characteristics, the above statement does not prove to be justified.

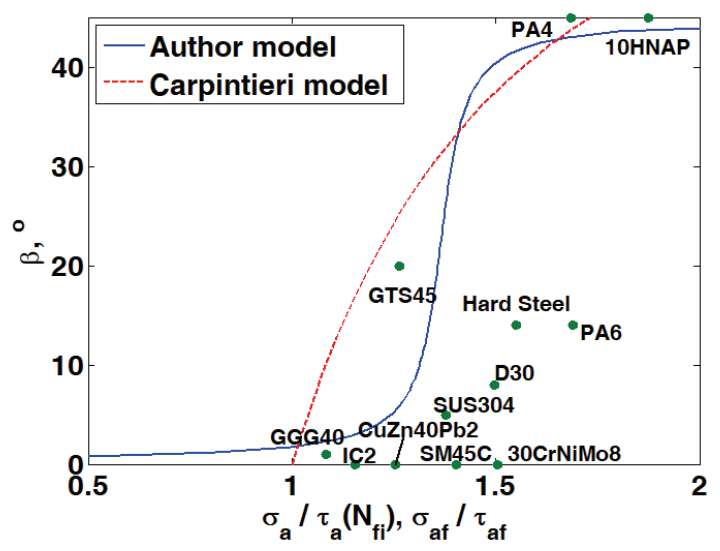

Fig. 2. The relationship between the angle $\beta$ and the parameter $\mathrm{B}_{2}$ (ratio of normal to shear stresses) according to different models

\section{Scatter analysis of fatigue life}

Every model needs to be verified. The analysis of the scatter bands of the estimated fatigue life can be applied for this purpose, both in relation to the proposed angle as well as depending on the ratio of the normal to shear stresses.

The analysis applied the values of the scatter bands for the particular materials just as it was calculated in [10] for angle $\beta$ in the range $<0^{\circ}, 45^{\circ}>$. Fig. 3 presents the methodology followed during the analysis of the proposed expression (6) with regard to the relation of angle $\beta$ and scatter $T$.

Assumption (1): The values of angles $\beta$ were calculated so that the scatter $\mathrm{T}$ is in the range $\left\langle T_{\min }, 1.1 T_{\min }>\right.$. On this basis, $\beta_{\text {opt }} \square<\beta_{\text {min }}, \beta_{\text {max }}>$.

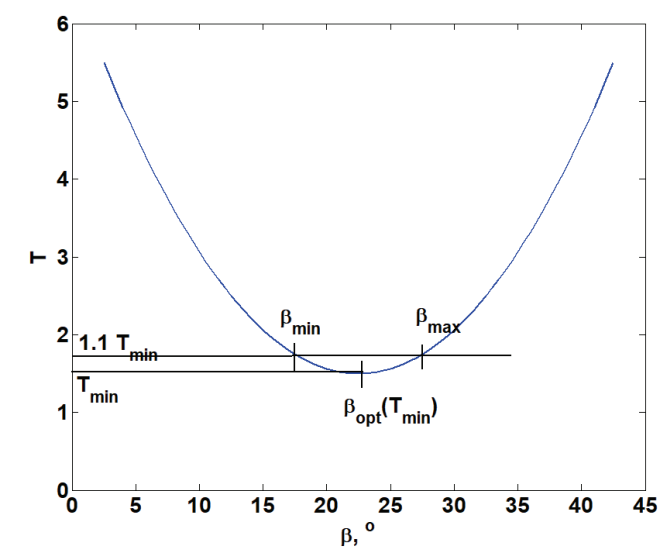

Fig. 3. Methodology of presented calculations - assumption 1

Fig. 4 illustrates the relation between scatter values $\mathrm{T}$ and the angle $\beta$ for aluminum alloy 2017A (known under the name PA6 [11]). 
Assumption (2): Ratio of normal to shear stresses is based on scatter for pure bending and pure torsion for selected materials in accordance with Fig. 5.

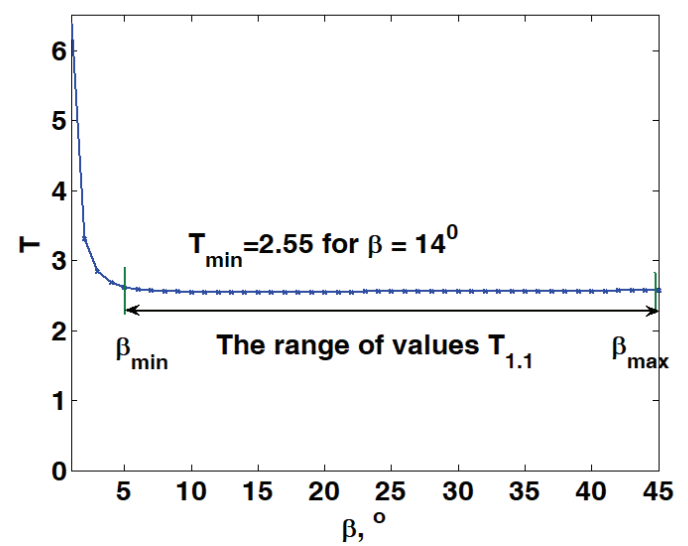

Fig. 4. Relationship between scatter values $T$ and the angle $\beta$ for PA6.

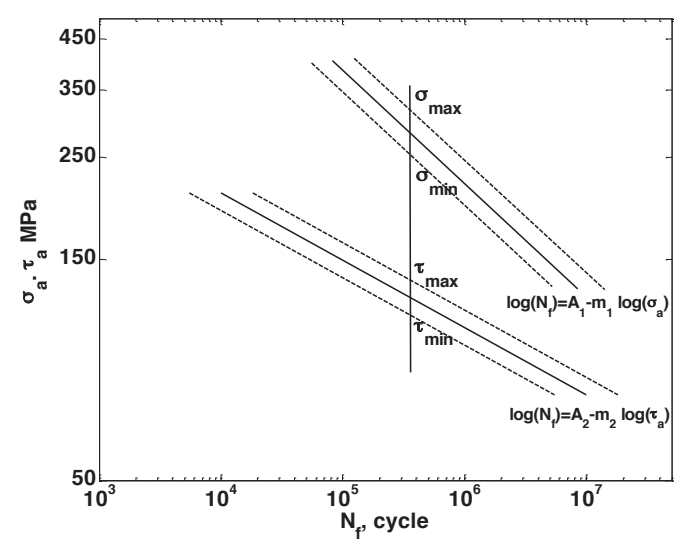

Fig. 5. Methodology of presented assumption 2.

The logarithmic dependence of the ratios of experimental and calculation strength should be used with the purpose of performing an adequate analysis of the fatigue life scatter.

A new method of determination the fatigue life scatter has been proposed by [9], where it was defined as the root mean square error

$$
E=\sqrt{\frac{\sum_{i=1}^{n} \log ^{2} \frac{N_{\text {exp }}}{N_{c a l}}}{n}}
$$

Therefore, the scatter can be determined as

$$
T=10 E
$$

where:

$$
B_{o p t}=\frac{\sigma_{a}\left(N_{f i}\right)}{\tau_{a}\left(N_{f i}\right)},
$$

$$
\begin{gathered}
B_{\text {max }}=\frac{\sigma_{\text {max }}\left(N_{f i}\right)}{\tau_{\text {min }}\left(N_{f i}\right)}, \\
B_{\text {min }}=\frac{\sigma_{\text {min }}\left(N_{f i}\right)}{\tau_{\text {max }}\left(N_{f i}\right)} .
\end{gathered}
$$

Fig. 6 contains the results of an experimental study for oscillatory bending and bilateral bending for the analyzed aluminum alloy. This chart also contains scatter bands calculated on the basis of relations 8 and 9 , which were applied for the calculations of $B_{o p t}, B_{\max }, B_{\min }$ parameters in accordance with relations (10-12). Table 1 contains a summary of the analyzed parameters for several materials selected from various material groups.

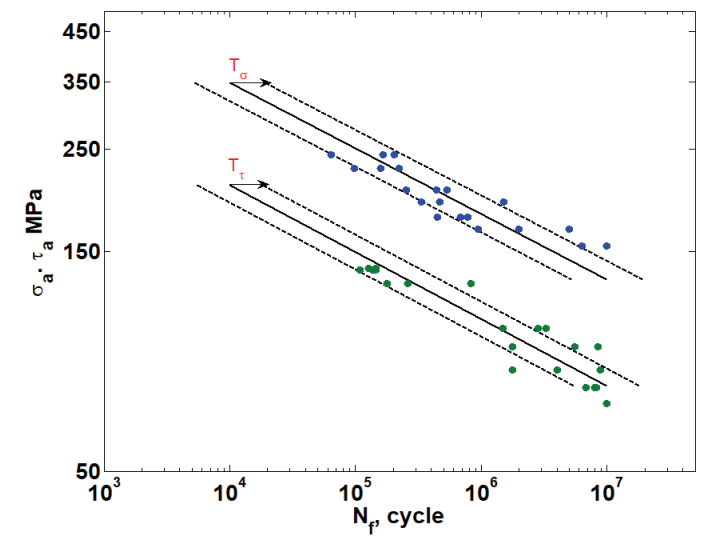

Fig. 6. Wöhler (Basquin) curves with scatter for pendulum bending and torsion for the analyzed alloy aluminum PA6.

Table 1. Analyzed parameters for selected materials.

\begin{tabular}{|c|c|c|c|c|c|}
\hline Material & $\boldsymbol{T}_{\boldsymbol{\sigma}}$ & $\boldsymbol{T}_{\boldsymbol{\tau}}$ & $\boldsymbol{B}_{\max }$ & $\boldsymbol{B}_{\min }$ & $\boldsymbol{B}_{\text {opt }}$ \\
\hline 10HNAP & 2.2 & 1.62 & 2.21 & 1.61 & 1.87 \\
\hline $\mathrm{CuZn40Pb2}$ & 1.49 & 1.44 & 1.37 & 1.15 & 1.25 \\
\hline PA6 & 1.91 & 1.82 & 2.02 & 1.41 & 1.69 \\
\hline GGG40 & 1.6 & 1.76 & 1.18 & 0.98 & 1.02 \\
\hline SUS304 & 1.62 & 1.2 & 1.5 & 1.22 & 1.38 \\
\hline
\end{tabular}

The results that were gained, were subsequently plotted onto the diagram representing the relation of angle $\beta$ depending on the ratio of normal to shear stresses (Fig. 7).

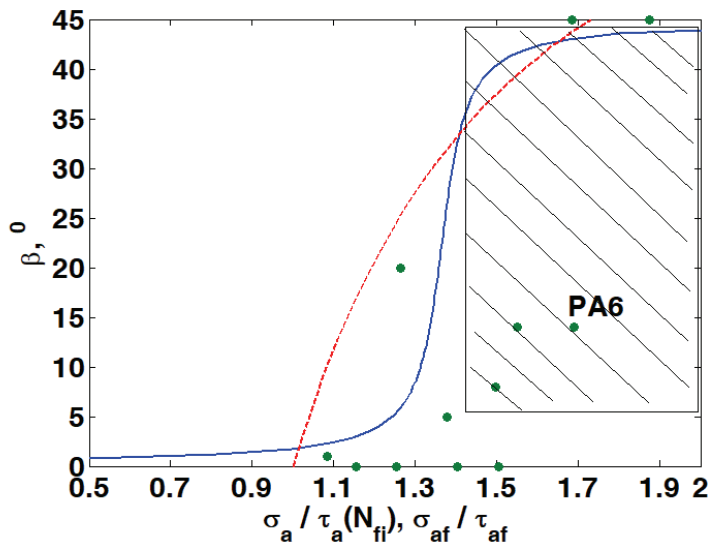

Fig. 7. Exemplary analysis of material PA6. 
Such an analysis was conducted with regard to all test materials. Fig. 8 presents the results of the analysis for selected materials: GTS45, GGG40, 10HNAP, SM45C. The hatched field is the area for which the results of the fatigue life are the same or differ insignificantly.

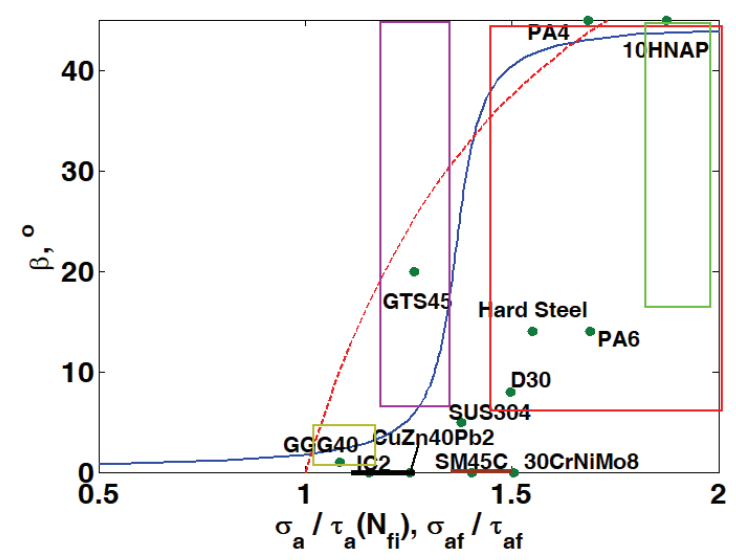

Fig. 8. Analysis results of selected materials.

On the basis of the analysis of Fig. 8, we can note that for some of the materials (e.g. GTS45, PA6), both models provide accurate results. The hatched fields involve both proposals for determination of the orientation angle of the critical plane. However, in some cases (e.g. SM45C, 10HNAP), the applicability fields only apply or are closer for the case of the proposition postulated by this author.

\section{Verification of the proposed criterion}

Fig. 9 presents comparison of the experimental fatigue life with the calculated fatigue life for Carpinteri's and Author's model. In this case the obtained results are satisfactory. A majority of the results are included into the scatter band of the coefficient 3.0. Such a system of points was easy to predict, as it was demonstrated by the analysis presented in Fig. 8.

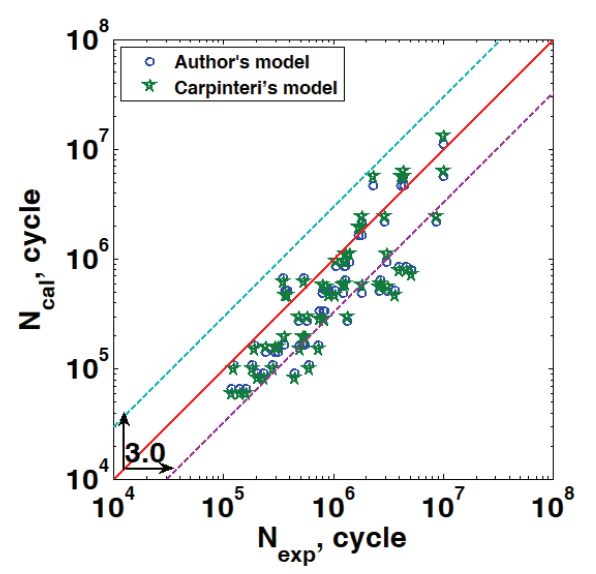

Fig. 9. Comparison of the calculated fatigue life $N_{c a l}$ with the experimental fatigue life $N_{\text {exp }}$ for aluminium alloy $2017 \mathrm{~A}$.

\section{Conclusions}

This paper was concerned with the verification of a new model applicable for the verification of the orientation angle of the critical plane. Under the assumption of two conditions, the study determined the applicability ranges of the proposed model. After calculations and analysis, the following conclusions can be stated:

1. The proposed verification confirmed the assumptions adopted with regard to the analyzed model.

2. The new expression for $\beta$ angle has a wider range than $<1, \sqrt{ } 3\rangle$.

3. The analysis performed in the paper involved the ratio of normal to shear stresses in the place of the traditional ratio of the fatigue limits. This is particularly important with regard to the materials with non-parallel characteristics.

4. Further analysis is needed with regard to successive materials in the future.

Project financed by the National Science Centre. Decision number: 2016/21/D/ST8/02007.

\section{References}

1. Ł. Pejkowski., D. Skibicki, Acta Mech. Sinica 32, 4, 696-709 (2016)

2. A. Carpinteri, C. Ronchei, A. Spagnoli, S. Vantadori, Theor. Appl. Fract. Mec. 73, 120-127 (2014)

3. M. Ciesla, G. Junak, A. Marek, Arch. Metal. Mater. 61, 1, 271-274 (2016)

4. G. Szala, B. Ligaj, Key Eng. Mater. 598, 231-236 (2014)

5. D. Skibicki, Ł. Pejkowski, Int. J. Fatigue 102, 18-36 (2017)

6. B. Zhong, W. Yanrong, W. Dasheng, W. Jialiang, Int. J. Fatigue 102, 241-251 (2017)

7. G. Stanfield, Discussion on "The strength of metals under combined alternating stresses", by H. Gough and H. Pollard. Proc. Institution of Mechanical Engineers, 131 (1935)

8. A. Carpinteri, A. Spagnoli, Int. J. Fatigue 23, 135145 (2001)

9. K. Walat, T. Łagoda, Int. J. Fatigue 67, 73-77 (2014)

10. A. Carpinteri M. Kurek, T. Łagoda, S. Vantadori Int. J. Fatigue 100, 512-520 (2017),

11. A. Niesłony, T. Łagoda, K. Walat, M. Kurek, Mat.wiss. U. Werkstofftech, 45 (10), 947-952 (2014) 